PHYSICAL REVIEW FLUIDS 2, 090502 (2017)

\title{
Progressive Monte Carlo rendering of atmospheric flow features across scales
}

\author{
Tobias Günther* \\ Computer Graphics Laboratory, ETH Zürich, Zürich, Switzerland \\ Alexander Kuhn \\ NVIDIA Advanced Rendering Center, Berlin, Germany \\ Hans-Christian Hege \\ Visual Data Analysis, Zuse Institute Berlin, Berlin, Germany \\ Markus Gross \\ Computer Graphics Laboratory, ETH Zürich, Zürich, Switzerland \\ Holger Theisel
}

Visual Computing Group, University of Magdeburg, Magdeburg, Germany

(Received 7 August 2017; published 29 September 2017)

\begin{abstract}
This paper is associated with a poster winner of a 2016 APS/DFD Milton van Dyke Award for work presented at the DFD Gallery of Fluid Motion. The original poster is available from the Gallery of Fluid Motion, https://doi.org/10.1103/APS.DFD.2016.GFM.P0030

DOI: 10.1103/PhysRevFluids.2.090502
\end{abstract}

\section{INTRODUCTION}

We demonstrate a technique to visualize multiple scales of atmospheric flow fields and the Lagrangian patterns therein, simulated by state-of-the-art simulation models for each scale. They provide insight into the structural differences and patterns that occur on each scale and highlight the complexity of flow phenomena in our atmosphere. For visualization, we employ unbiased and consistent Monte Carlo rendering to avoid discretization of finite-time Lyapunov exponent fields.

High-resolution climate data can improve existing weather prediction and reanalysis capabilities. High-resolution numerical simulations of atmospheric flows help scientists to understand aerodynamic processes and to visualize the structural flow patterns across scales.

\section{METHOD}

The finite-time Lyapunov exponent (FTLE) is an indicator for Lagrangian coherent structures. We use the gradient $\nabla \phi=\frac{\partial \phi}{\partial \mathbf{x}}$ of the flow map $\phi_{t}^{\tau}(\mathbf{x})$ to define FTLE for start time $t$ and duration $\tau$ as [1]

$$
\Lambda(\mathbf{x}, t, \tau)=\frac{1}{|\tau|} \ln \sqrt{\lambda_{\max }\left[(\nabla \phi)^{\mathrm{T}} \nabla \phi\right]}
$$

This field is visualized by mapping the values via transfer functions to an extinction coefficient $\sigma_{t}$ and a scattering albedo, casting this into a light transport problem in a heterogeneous medium [2].

\footnotetext{
*tobias.guenther@inf.ethz.ch

Published by the American Physical Society under the terms of the Creative Commons Attribution 4.0 International license. Further distribution of this work must maintain attribution to the author(s) and the published article's title, journal citation, and DOI.
} 


\section{GÜNTHER, KUHN, HEGE, GROSS, AND THEISEL}
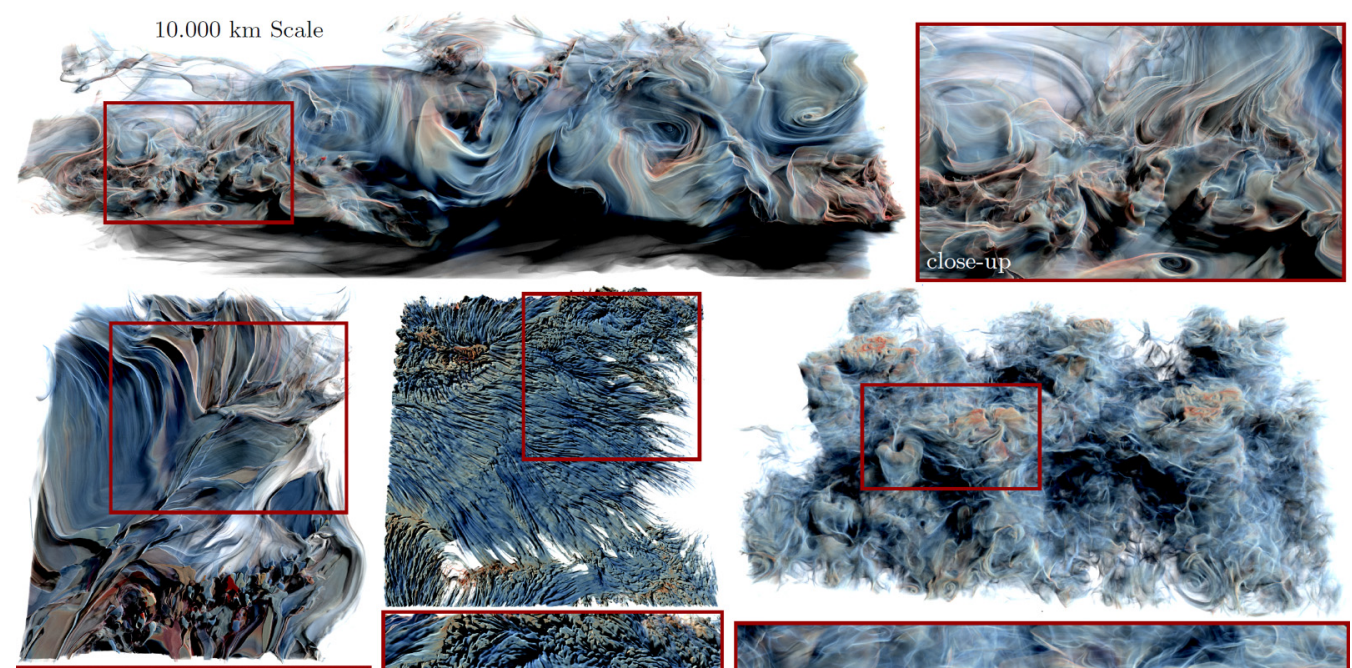

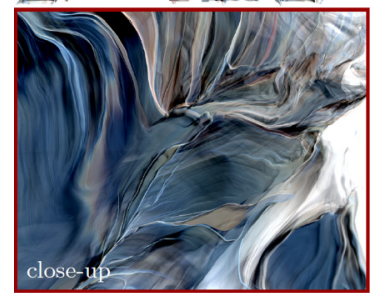

$1.000 \mathrm{~km}$ Scale

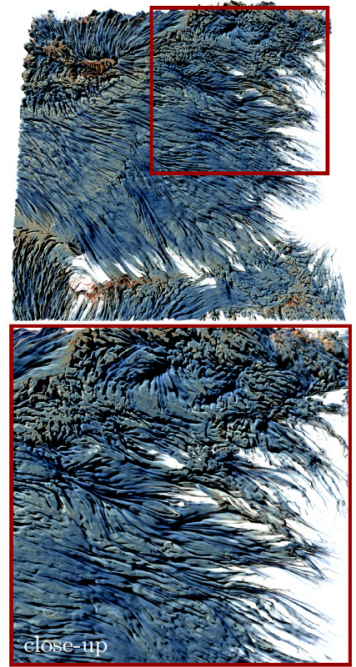

$100 \mathrm{~km}$ Scale

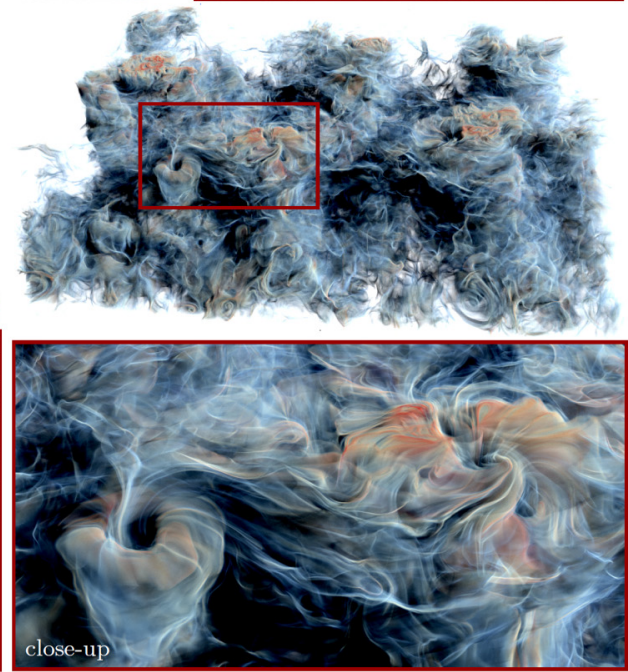

$10 \mathrm{~km}$ Scale

FIG. 1. Lagrangian visualizations of atmospheric air flows on different scales. See the original poster at https://doi.org/10.1103/APS.DFD.2016.GFM.P0030 (CC BY-NC 4.0).

The radiance $L$ coming in with direction $\vec{\omega}$ on a sensor at $\mathbf{x}$ is found by collecting the incoming radiance $L_{i}$ that is scattered with coefficient $\sigma_{s}$ towards the viewer, accounting for transmittance $T_{r}$ :

$$
L(\mathbf{x} \leftarrow \vec{\omega})=\int_{0}^{d} T_{r}\left(\mathbf{x}_{s} \leftrightarrow \mathbf{x}\right) \sigma_{s}\left(\mathbf{x}_{s}\right) L_{i}\left(\mathbf{x}_{s} \leftarrow \vec{\omega}\right) d s,
$$

for which we use Delta tracking [3]. The resulting visualization highlights areas of strong repelling behavior. The ridge surfaces indicate material structures, govern the advection of trace gases (such as $\mathrm{CO}_{2}$ or $\mathrm{SO}_{2}$ ), guide temperature diffusion, and influence cloud formation.

\section{RESULTS}

\section{A. The 10.000-km scale (global scale)}

Figure 1 (top) shows a reanalysis simulation [4] of the northern hemisphere (data from the European Centre for Medium-Range Weather Forecasts), including the North American land surface, the North Atlantic Ocean, and the European land mass. Here, atmospheric flows are shown on a large scale. The exchange of energy and trace gases is related to the FTLE field, which reveals characteristic flow patterns in the atmosphere. A closeup highlights the North American land surface. In particular, the turbulent structure of vortices and stream-like features are emphasized.

\section{B. The 1.000-km scale (synoptic scale)}

The COSMO-DE reanalysis simulation displays the air flow over central Europe. The model is currently in use by the German weather service. At the synoptic scale, flow patterns are strongly 
influenced by global-scale features, land-sea interaction, and orography. This is apparent in Fig. 1 (bottom, left) when comparing flow structures over the alpine region (South) and the northern coastline. Here, flow separation takes place at the central region, caused by a rain front passing from southwest to northeast. The closeup depicts the northeastern part of Germany.

\section{The 100-km scale (mesoscale)}

At the mesoscale, a semi-idealized large eddy simulation (LES) is shown for the area around Jülich, Germany, with a spatial resolution of $50 \mathrm{~m}$ [5]. The simulation uses boundary forcing from the COSMO-DE model. At this scale, convective plumes and updraft cells can be resolved and simulated directly. Figure 1 (bottom, center) shows a convective cell at the top left, which creates a strong, separating updraft with visible intake around the cell. The closeup shows detailed features compared to the synoptic simulations.

\section{The 10-km scale}

The smallest scale displays a cloud-topped boundary layer simulation, created using the UCLALES model [6]. The simulation contains a cloud resolving domain with 25-m spatial resolution under idealized conditions: It uses double-periodic boundary conditions and homogeneous surface forcing, while large-scale information is taken from the COSMO-DE model. Similar to the mesoscale, the purpose is to determine a subscale parametrization of synoptic simulations. Figure 1 (bottom, right) shows convective flow patterns, e.g., separation patterns visible in the FTLE field.

\section{ACKNOWLEDGMENTS}

This work was partially supported by DFG Grant No. TH 692/8-1 and by BMBF Grant No. 01LK1213C.

[1] G. Haller, Distinguished material surfaces and coherent structures in three-dimensional fluid flows, Phys. D (Amsterdam, Neth.) 149, 248 (2001).

[2] T. Günther, A. Kuhn, and H. Theisel, MCFTLE: Monte Carlo rendering of finite-time Lyapunov exponent fields, Computer Graphics Forum 35, 381 (2016).

[3] E. R. Woodcock, T. Murphy, P. J. Hemmings, and T. C. Longworth, Techniques used in the GEM code for Monte Carlo neutronics calculations in reactors and other systems of complex geometry, in Proceedings of the Conference on the Application of Computing Methods to Reactor Problems, ANL-7050, Argonne National Laboratory (1965), pp. 557-579.

[4] D. P. Dee, S. M. Uppala, A. J. Simmons, P. Berrisford, P. Poli, S. Kobayashi, U. Andrae, M. A. Balmaseda, G. Balsamo, P. Bauer et al., The ERA-interim reanalysis: Configuration and performance of the data assimilation system, Quart. J. R. Meteorol. Soc. 137, 553 (2011).

[5] R. Heinze, C. Moseley, L. N. Böske, S. K. Muppa, V. Maurer, S. Raasch, and B. Stevens, Evaluation of large-eddy simulations forced with mesoscale model output for a multi-week period during a measurement campaign, Atmos. Chem. Phys. 17, 7083 (2017).

[6] B. Stevens, C.-H. Moeng, A. S. Ackerman, C. S. Bretherton, A. Chlond, S. de Roode, J. Edwards, J.-C. Golaz, H. Jiang, M. Khairoutdinov et al., Evaluation of large-eddy simulations via observations of nocturnal marine stratocumulus, Monthly Weather Rev. (AMS) 133, 1443 (2005). 